\title{
Establishing and transducing cell polarity: common themes and variations
}

Daniel St Johnston

The Gurdon Institute and the Department of Genetics

\section{Address:}

University of Cambridge

Tennis Court Rd

Cambridge CB2 1QN

Email: d.stjohnston@gurdon.cam.ac.uk

\begin{abstract}
All cells in vivo have a primary axis of polarity that controls many aspects of their behaviour, such as the direction of protein secretion and signalling, the orientation of cell division and directed cell movement and morphogenesis. Cell polarise in response to extracellular cues or intracellular landmarks that initiate a signal transduction process that establishes complementary cortical domains of conserved polarity factors. These cortical domains then transmit this polarity to the rest of the cell by regulating the organisation of the cytoskeleton and membrane trafficking systems. Here I review work over the past couple of years that has elucidated many key features of how polarity is established and transduced in different systems, but has also revealed unexpected variations in polarity mechanisms depending on context.
\end{abstract}

\section{Introduction}

Cellular shape and architecture must be tightly regulated in vivo, so that the cells perform their functions in the correct place and make the appropriate connections with each other to form a multicellular organism. The first step in establishing this architecture is the creation of a primary axis of polarity, whose orientation is 
determined by local cues. This polarity then propagates to the rest of the cell by regulating the organisation of the cytoskeleton and the direction of intracellular trafficking pathways. Some cells polarise only transiently. For example, migrating cells establish a front-rear polarity that changes with their direction of movement [1], while T-lymphocytes polarise secretion towards the immunological synapse to kill their targets and then repolarise to move towards the next target [2]. This review will focus, however, on cells that establish a stable axis of polarity, such as the apicalbasal axis of epithelial cells or the inside-out polarity of blastomeres, but excluding neuronal polarity, as this is a huge field on its own that has recently been reviewed elsewhere $[3,4]$. The first section of the review will consider recent advances in our understanding of how polarity is established in different contexts, which have revealed both a surprising plasticity in polarity mechanisms, as well as some recurring themes and common components. The second section will then focus of the conserved set of cortical polarity proteins that maintain and propagate polarity in the most cells, focusing on work that has begun to elucidate how these factors interact to maintain polarity and how they transduce this asymmetry to organise membrane domains, target secretion and polarise the cytoskeleton.

Yeast polarity The budding yeast, $S$. cerevisiae, has provided a paradigm for understanding and modelling how cells polarise. Yeast cells can polarise in response to mating pheromones or internal landmarks that differ between haploid and diploid cells. All of these cues result in the formation of a single zone of active Cdc42-GTP, 
which then activates several downstream pathways to define the bud tip [5]. In the absence of all of these cues, however, cells still polarise towards a single randomlypositioned focus of active Cdc42-GTP. This is thought to occur through a positive feedback loop that amplifies an initial stochastic asymmetry in Cdc42 activation, in which the Cdc42-GTP effector the Cla1/Bem1 complex recruits and activates the Cdc42 GTP/GDP exchange factor Cdc24, which then activates more Cdc42 locally[6,7]. This needs to be coupled with the faster exchange between the membrane and the cytoplasm of Cdc42-GDP than Cdc42-GTP, and a negative feedback process that prevents the unlimited growth of the focus [7-10]. There must also be a mechanism to prevent multiple Cdc42-GTP foci forming, which is likely to occur by competition between foci for limited cytoplasmic components [11]. While this model can explain the properties of the system, the final proof that it is correct has come from elegant experiments using optogenetics to recruit Bem1 or Cdc24 to the cortex $\left[12^{* *}\right]$. Localising either protein to a region of the cell cortex is sufficient to activate a positive feedback loop that recruits the endogenous factors to the site of light-induced localisation, defining where Cdc42-GTP accumulates and where the bud tip forms. Thus, these simple positive and negative feedback loops provide a robust mechanism to polarise growth towards a single point, as predicted by computer simulations [13].

Similar Cdc42 feedback loops may play a role in polarising animal cells. For example, knock out of Cdc42 in Ls174T:W4 cells (an enterocyte cell line that polarises as single cells when Lkb1 is activated) leads to cells with multiple apical domains [14*]. Thus, Cdc42 is not essential for apical domain formation in this system, but ensures that only one domain forms per cell. This also requires the lipid 
flippase ATP8B1, which acts to prevent the diffusion of active Cdc42 by increasing the levels of phosphatidylserine in the inner leaflet of the plasma membrane.

In fission yeast, positive and negative feedback loops also control Cdc42 activation to polarise growth, although this is normally restricted to the cell ends by a microtubuledependent mechanism that deposits clusters of Tea1 and Tea4 at the poles $[5,15,16]$. However, it was recently found that cells exiting from starvation polarise by a Cdc42independent mechanism, in which sterol-rich membrane domains define growth sites that become restricted to the poles through the microtubule/Tealp pathway [17*].

\section{C. elegans anterior-posterior polarity}

Most animal cells do not polarise towards a single focus like budding yeast, but instead form complementary cortical domains that mark opposite sides of the cell. How this polarity is established is best understood in C. elegans, where the anteriorposterior axis is defined by the polarisation of the one cell embryo into complementary domains that are marked by the localisation of PAR-3, PAR-6 and atypical protein kinase $\mathrm{C}$ (PKC-3) to the anterior cortex and PAR-1 and PAR-2 posteriorly. The anterior PAR proteins are initially localised to the entire cortex and symmetry is broken by the entry of the sperm centrosome, which triggers a contraction of the acto-myosin cortex towards the anterior $[18,19]$. The anterior PARs move by advection with the contraction, allowing the posterior PAR proteins to colonise the posterior cortex, and these complementary cortical domains are then maintained by mutual antagonism between the anterior and posterior PAR proteins. 
Recent work has begun to reveal the mechanisms that underlie this mutual antagonism. Anterior aPKC activity excludes the posterior factors, PAR-1, PAR-2 and the Cdc42-GTPase activating protein (CDC42-GAP), CHIN-1, while PAR-1 and CHIN-1 act redundantly to prevent aPKC activation posteriorly [20**]. PAR-1 is thought to directly phosphorylate PAR-3 to prevent is oligomerisation, which is essential for the efficient recruitment of aPKC to the cortex $[21,22]$. CHIN-1, on the other hand, inactivates CDC42, thereby preventing it from activating aPKC through binding to the PAR-6/aPKC complex [23]. Both PAR-3 and CHIN-1 form clusters at the cortex that are inhibited by Par- 1 and aPKC respectively, and computational modelling suggests that this makes the system hypersensitive to small changes in PAR-6/aPKC activity, leading to a sharper boundary between the anterior and posterior Par domains[20**].

PAR3 clustering also plays a critical role in coupling the movement of the anterior PAR proteins to the cortical contraction. Elegant studies using single embryo biochemistry and single molecule imaging have shown that PAR-3 forms large oligomers during polarity establishment that are required for the efficient cortical localisation of PAR-3, the recruitment of the PAR-6/aPKC complex and the movement of the entire complex with the actomyosin contraction[24**, 25**,26*]. At the onset of the maintenance phase, Polo kinase phosphorylates the PAR-3 oligomerisation domain to inhibit clustering. This results in a reduced association of PAR-6/aPKC with PAR-3, leading to the formation of a diffusible complex of Cdc42, PAR-6 and active aPKC that acts to exclude the posterior PARs. Structural analysis indicates that PAR-3 inhibits the kinase activity of aPKC [27] This suggests that PAR-6/aPKC cycles between two states: an inactive complex with PAR-3, which 
recruits PAR-6/aPKC to the membrane and mediates its anterior localisation and an active complex with Cdc42-GTP that phosphorylates the posterior PARs to maintain polarity.

\section{Apical-basal polarity in blastomeres:}

In C.elegans, apical-basal polarity emerges at the four-cell stage, when Cdc42, PAR3, PAR-6 and aPKC localise to the contact-free, outer surface of the blastomeres and PAR-1 and PAR-2 localise to areas of cell-cell contact [28,29]. This process is driven by the recruitment of the $\mathrm{Cdc} 42-\mathrm{GAP}, \mathrm{PAC}-1$, to cell-cell contacts, thereby restricting Cdc42 activity to the free apical surface [30]. Recent results have revealed that Ecadherin-mediated cell adhesion (HMR-1 in C. elegans) plays an instructive role in this process by recruiting PAC-1 to cell contacts through JAC-1 (p120-catenin) and PICC-1 [31*]. Interestingly, a large scale protein interaction map found that PAC-1 also binds to PAR-6 and mutation of this PAR- 6 binding domain disrupted polarity establishment [32*]. Since PAR-6 localises to the opposite domain to PAC-1 once the cells are polarised, it is unclear why this interaction is essential, but one possibility is that PAR-6 helps to deliver active Cdc42-GTP to PAC-1, so that it can be inactivated at cell contacts through the hydrolysis of GTP.

Early mouse blastomeres show a similar outside-in polarisation starting from the 8cell stage, with Par-6, Ezrin and aPKC localising to the contact-free apical surface and Lgl to the sites of cell adhesion[33]. Apical factors localise around the entire cell cortex of most cells in E-cadherin null embryos, suggesting that cell contact excludes apical factors by a similar mechanism to C. elegans blastomeres. This cannot be the only mechanism to establish polarity, however, as isolated blastomeres from 8-cell 
embryos polarise spontaneously in the absence of cell contact: they then proceed to divide asymmetrically to produce one polarised cell that becomes trophectoderm and engulfs the other apolar daughter cell, which expresses inner cell mass markers [34**]. Furthermore, apolar cells can be induced to polarise by touching them with a polymethyl-methacrylate bead, and this procedure works efficiently even if the bead is uncoated and the cells lack E-cadherin. Thus, mouse blastomeres can polarize in the absence of cell adhesion and their polarity can be oriented by solely mechanical cues.

\section{Polarising epithelial cysts:}

De novo polarity emerges in a quite different way when single MDCK cells are cultured in Matrigel $[35,36]$. Apical markers, such as Podocalyxin and Crumbs 3, initially localise all round the plasma membrane, but when the cell divides, these are trancytosed via Rab11-positive recycling endosomes to an apical membrane initiation site (AMIS) that forms around the midbody and is marked by PAR-3, components of the Exocyst and Cingulin [37]. Fusion of these transcytotic vesicles at the AMIS generates an apical domain and lumen at the site of cytokinesis, giving rise to polarised minicysts. Although a variety of Rab GTPases are required for the trafficking and fusion of these apical vesicles, Rab35 seems to play a particularly important role $\left[38,39^{* *}\right]$. It localizes to the cleavage furrow before the AMIS forms and binds directly to the cytoplasmic tail of Podocalyxin to capture vesicles around the midbody [39**]. Furthermore, knock-down of Rab35 or mutation of its Podocalyxin binding site gives rise to cysts with inverted polarity. This is not the only mechanism that positions the AMIS, however, as Cingulin is recruited to site of cytokinesis by direct binding to the midbody microtubules, where it recruits Rab11positive vesicles by binding to the Rab11 interacting protein FIP5 [37,40**]. It is not clear whether Rab35 and Rab11/Cingulin function in the same pathway or in parallel, 
but it seems likely that multiple mechanisms couple cell division to polarity in this system.

\section{Polarity Transduction}

Once polarity is established, it needs to be propagated to the rest of the cell. In epithelial cells, for example, the initial polarization must be elaborated to specify where the adherens junctions form to link adjacent cells together; where the occluding junctions (tight junctions and septate junctions) are positioned to create the barrier to paracellular diffusion; specific cargoes must be directed to either the apical or the basolateral membranes for polarised secretion; and the actin and microtubule cytoskeletons must be organized to direct intracellular transport and provide mechanical support [41].

In most epithelial cells, polarity is maintained and transduced by an expanded repertoire of the same PAR proteins that define polarity in the C. elegans zygote: the apical domain is marked by Cdc42, Par-6, aPKC and the epithelial-specific polarity proteins, Crumbs, Stardust (PALS) and Patj, the apical junction is defined by the localisation of Par-3/Bazooka and the rest of the lateral domain by Scribble, Discs large (Dlg) and Lethal (2) giant larvae (Lgl) and Par-1 [42]. Polarity maintenance depends on mutual antagonism between apical and lateral factors, and recent work has elucidated the mechanism behind the mutually-exclusive membrane localisation of aPKC and $\operatorname{Lgl}\left[43^{* *}, 44^{* *}\right]$. These studies revealed that aPKC phosphorylates several serines in a basic domain of Lgl that binds membrane phospholipids, introducing negative charges that prevent lipid binding, thereby excluding Lgl from the apical region. Furthermore, aPKC restricts several other proteins to the basolateral domain by a similar mechanism, including Miranda, which targets cell fate determinants 
basally during the asymmetric divisions of Drosophila neuroblasts.

Polarity factors can also regulate the localisation of other proteins by altering the membrane lipid composition. In many polarized epithelia, Phosphatidylinositol 4,5 bisphosphate (PIP2) is enriched apically and Phosphatidylinositol 3, 4,5 trisphosphate (PIP3) basolaterally [45]. Par-3 localises to the apical junction that forms the boundary between these domains and has been proposed to contribute to the differential distribution of PI2 and PIP3 by recruiting PTEN, which catalyses the conversion of PIP3 to PIP2 and PI3 kinase, which converts PIP2 to PIP3. This has now been elegantly demonstrated by using optogenetics to mislocalise Par-3 to the apical domain [46**]. This induces PIP3 production and the activation of Rac to disrupt apical domain identity, a mechanism that is exploited by the bacterium, Neisseria meningitidis, to cross endothelial barriers.

\section{Cadherin targeting}

One of the hallmarks of epithelial polarity is the localisation of E-cadherin to adherens junctions that hold the epithelial cells together. During Drosophila cellularisation, Par-3 (Bazooka) plays a key role in localising Cadherin to the apical adherens junctions, in part by coupling it to dynein-mediated microtubule transport [47]. It has recently been found that Par-3 is also required for Cadherin localisation in the mammary epithelium, in this case by targeting its exocytosis to the junctional domain $\left[48^{* *}\right]$. A C-terminal lysine rich domain of Par-3 directly recruits the exocyst complex, which is required for the fusion of E-cadherin-containing vesicles with the plasma membrane. E-cadherin is not therefore not delivered to the junctions in Par-3 mutant cells and accumulates in intracellular vesicles. Lgl, on the other hand, 
regulates $\mathrm{N}$-cadherin localisation in the mouse brain by enhancing its internalisation from the lateral membrane through direct binding of Lgl's WD40 repeats to the cytoplasmic tail of E-cadherin [49**]. Thus, it is attractive to propose that Par-3 and Lgl collaborate to position Adherens junctions by removing nonjunctional Cadherins from the lateral membrane and targeting their recycling to the junctional domain.

\section{Polarity transduction to the cytoskeleton}

Cell polarisation has profound effects on the organisation of the actin and microtubule cytoskeletons. Actin is mainly organized locally from sites of cell substrate and cellcell adhesion that are themselves regulated by the polarity system, but can also be directly organized by polarity factors. For example, it has recently been shown that the Cdc42-dependent activation of the myosin light chain kinase, MRCK induces apical acto-myosin contractility, which plays an important role in the expansion of the apical domain and microvilli formation $\left[50^{*}\right]$. The microtubule cytoskeleton is organized on a larger scale than actin, often spanning the entire cell and is tightly coupled to cortical polarity. The last few years have provided several important insights into how polarity system and microtubules interact.

Polarity plays a key role in microtubule organisation during the process of oriented cell division. This has been best characterized during the asymmetric divisions of Drosophila neuroblasts, where apical Baz/Par-3 recruits Inscuteable, which in turn recruits the spindle orientation machinery of Pins/LGN, Mud/NUMA, Dlg and Dynein to pull on astral microtubules and orient the spindle along the apical-basal axis [51]. Baz/Par-3 plays a similar role in orienting the asymmetric divisions of the Drosophila male germline stem cell, but in this case Baz/Par-3 recruits the centrosome by an unknown mechanism that does not involve Inscuteable, but requires 
Baz/Par-3 phosphorylation by Par-1 [52**]. This activity can also be observed in Drosophila embryos knocked down for aPKC, where centrosomes are recruited to patches of Baz/Par-3 protein in a Par-1-dependent manner [53*]. An interesting variation on this theme occurs in sensory organ precursor cells in the fly notum, where the spindle aligns along the anterior-posterior rather than the apical-basal axis, because Baz/Par3 is recruited to posterior cortex under the control of the planar cell polarity (PCP) pathway[54]. This depends on the SOP-specific expression of the RASSF protein Meru, which associates with the Frizzled/Disheveled PCP complex to recruit Baz/Par-3 to the posterior [55**]. This is an unusual example where the canonical apical-basal polarity factors lie downstream of the PCP pathway, but maintain their role in organizing the microtubules of the spindle.

In contrast to asymmetric stem cell divisions, epithelial cells always orient their spindles in the plane of the epithelium, which requires positional cues from the lateral cortex. The lateral polarity factor, Dlg, recruits Pins/NUMA to orient spindles in Drosophila follicle cells and the chick neural tube [56,57]. This is not the case in all epithelia, however, as Pins is dispensable for spindle orientation in the Drosophila wing disc $[58,59]$. Instead, the spindle is oriented by pulling forces from tricellular junctions that depend on the recruitment of Mud and dynein. This mechanism provides a memory of the interphase cell shape and allows the cells to orient their spindles along their long axes, even though the cells have rounded up on entering mitosis.

The centrosome moves to the apical surface of epithelial cells in interphase, and in the case of ciliated epithelia, the mother centriole then becomes the basal body that nucleates the primary cilium [60]. The apical positioning of the centrosome depends 
on Par-3 and the actin cytoskeleton, but the underlying mechanisms are unknown $[61,62]$. Centrosome positioning seems to be intimately linked with epithelial polarity, however, as the movement of the centrosome to a more central position is one of the earliest events in epithelial to mesenchymal transitions [63*].

Although the centrosome is the main microtubule organizing centre (MTOC) in mitotic cells, many interphase or postmitotic cells organize microtubule arrays from noncentrosomal MTOCs at the cortex [64]. For example, epithelial cells form apicalbasal arrays of microtubules that play an important role in polarized trafficking. These arrays can be generated either by anchoring pericentrosomal material, including the microtubule-nucleating $\gamma$-tubulin ring complex at the apical cortex, coupled with microtubule anchoring by Ninein $[65,66]$, or more commonly through the apical recruitment of the microtubule minus end stabilizing complex composed of Shot/ACF7, Patronin/CAMSAP2-3 and Katanin that captures and stabilize minus ends, so that new microtubules can grow from them [67-70]. What is much less well understood is how these ncMTOCs are localised apically, although apical $\beta_{\mathrm{H}}$-spectrin has been proposed to play a role [69*]. Some insight to how the polarity systems can regulate ncMTOC localisation comes from studies on the Drosophila oocyte, where Shot/Patronin noncentrosomal MTOCs form along the anterior and lateral cortex but are excluded from the posterior, resulting in the formation of a polarised microtubule network that directs the localisation of the axis determinants [68**]. In this case, oocyte polarity is coupled to microtubule organisation by PAR-1, which localises to the posterior cortex and excludes Shot

\section{Conclusion}

The last couple of years have seen a great deal of progress in our understanding of 
how cells polarise and how this polarity is transduced, but this has also revealed an unexpected diversity of mechanisms. Although the canonical polarity factors, such as Par-3, aPKC and Cdc42 play crucial roles in many of these processes, their partners and functions are context-dependent. Furthermore, it now becoming clear that some cells can polarize in the absence of these factors and that these proteins have roles that are unrelated to polarization[71,72]. Thus, the challenge for the future will be to understand how polarity mechanisms vary between cell-types and whether there are alternative polarity pathways that operate in specific contexts.

1. Devreotes PN, Bhattacharya S, Edwards M, Iglesias PA, Lampert T, Miao Y: Excitable Signal Transduction Networks in Directed Cell Migration. https://doi.org/10.1146/annurev-cellbio-100616-060739 2016, doi:10.1146/annurev-cellbio-100616-060739.

2. la Roche de M, Asano Y, Griffiths GM: Origins of the cytolytic synapse. Nat. Rev. Immunol. 2016, 16:421-432.

3. Bentley M, Banker G: The cellular mechanisms that maintain neuronal polarity. Nat. Rev. Neurosci. 2016, 17:611-622.

4. Rolls MM, Jegla TJ: Neuronal polarity: an evolutionary perspective. $J \operatorname{Exp}$ Biol 2015, 218:572-580.

5. Chiou J-G, Balasubramanian MK, Lew DJ: Cell Polarity in Yeast. Annu Rev Cell Dev Biol 2017, 33:annurev-cellbio-100616-060856.

6. Kozubowski L, Saito K, Johnson JM, Howell AS, Zyla TR, Lew DJ: Symmetry-breaking polarization driven by a Cdc42p GEF-PAK complex. Curr Biol 2008, 18:1719-1726.

7. Rapali P, Mitteau R, Braun C, Massoni-Laporte A, Ünlü C, Bataille L, Arramon FS, Gygi SP, McCusker D: Scaffold-mediated gating of Cdc42 signalling flux. Elife 2017, 6:e25257.

8. Kuo C-C, Savage NS, Chen H, Wu C-F, Zyla TR, Lew DJ: Inhibitory GEF phosphorylation provides negative feedback in the yeast polarity circuit. Curr Biol 2014, 24:753-759.

9. Howell AS, Jin M, Wu C-F, Zyla TR, Elston TC, Lew DJ: Negative feedback enhances robustness in the yeast polarity establishment circuit. Cell 2012, 149:322-333. 
10. Woods B, Lai H, Wu C-F, Zyla TR, Savage NS, Lew DJ: Parallel ActinIndependent Recycling Pathways Polarize Cdc42 in Budding Yeast. Curr Biol 2016, 26:2114-2126.

11. Wu C-F, Chiou J-G, Minakova M, Woods B, Tsygankov D, Zyla TR, Savage NS, Elston TC, Lew DJ: Role of competition between polarity sites in establishing a unique front. Elife 2015, 4:e11611.

12**. Witte K, Strickland D, Glotzer M: Cell cycle entry triggers a switch between two modes of Cdc42 activation during yeast polarization. Elife 2017, 6:e26722.

Optogenetic recruitment of $\mathrm{Cdc} 24$ or Bem 1 to a region of the yeast membrane is sufficient to induce a persistent focus of active Cdc42-GTP that defines the future bud site, proving that positive feedback through these factors stabilises the polarity site.

13. Chau AH, Walter JM, Gerardin J, Tang C, Lim WA: Designing synthetic regulatory networks capable of self-organizing cell polarization. Cell 2012, 151:320-332.

14*. Bruurs LJM, Donker L, Zwakenberg S, Zwartkruis FJ, Begthel H, Knisely AS, Posthuma G, van de Graaf SFJ, Paulusma CC, Bos JL: ATP8B1-mediated spatial organization of $\mathrm{Cdc42}$ signaling maintains singularity during enterocyte polarization. $J$ Cell Biol 2015, 210:1055-1063.

Loss of the lipid flippase ATP8B1 increases Cdc42 mobility at the membrane, leading to apical domain expansion and the formation of multiple apical domains.

Multiple apical domains are also observed in $\mathrm{Cdc} 42$ null cells, indicating that $\mathrm{Cdc} 42$ is required to suppress extra apical domains, but is not required for their formation.

15. Bendezú FO, Vincenzetti V, Vavylonis D, Wyss R, Vogel H, Martin SG: Spontaneous Cdc42 Polarization Independent of GDI-Mediated Extraction and Actin-Based Trafficking. PLoS Biol 2015, 13:e1002097.

16. Martin SG, Arkowitz RA: Cell polarization in budding and fission yeasts. FEMS Microbiol. Rev. 2014, 38:228-253.

17*. Makushok T, Alves P, Huisman SM, Kijowski AR, Brunner D: Sterol-Rich Membrane Domains Define Fission Yeast Cell Polarity. Cell 2016, 165:1182-1196.

Fission yeast cells recovering from starvation polarise growth towards Sterol-rich membrane domains that become restricted to the poles by the Teal system. Unlike other fission yeast polarity systems, the cells can polarise without Cdc42.

18. Cheeks RJ, Canman JC, Gabriel WN, Meyer N, Strome S, Goldstein B: C. elegans PAR proteins function by mobilizing and stabilizing asymmetrically localized protein complexes. Curr Biol 2004, 14:851-862. 
19. Munro E, Nance J, Priess JR: Cortical flows powered by asymmetrical contraction transport PAR proteins to establish and maintain anteriorposterior polarity in the early C. elegans embryo. Dev Cell 2004, 7:413-424.

20**. Sailer A, Anneken A, Li Y, Lee S, Munro E: Dynamic Opposition of Clustered Proteins Stabilizes Cortical Polarity in the C. elegans Zygote. Dev Cell 2015, 35:131-142.

This study shows that polarity of the C. elegans zygote is maintained by antagonism between anterior PKC-3 (aPKC) and two redundant posterior factors, PAR-1 and CHIN-1. Clustering of CHIN-1 is essential for the formation of a sharp boundary between the anterior and posterior domains.

21. Benton R, St Johnston D: Drosophila PAR-1 and 14-3-3 inhibit Bazooka/PAR-3 to establish complementary cortical domains in polarized cells. Cell 2003, 115:691-704.

22. Motegi F, Zonies S, Hao Y, Cuenca AA, Griffin E, Seydoux G: Microtubules induce self-organization of polarized PAR domains in Caenorhabditis elegans zygotes. Nat Cell Biol 2011, 13:1361-1367.

23. Atwood SX, Chabu C, Penkert RR, Doe CQ, Prehoda KE: Cdc42 acts downstream of Bazooka to regulate neuroblast polarity through Par-6 aPKC. J Cell Sci 2007, 120:3200-3206.

24**. Dickinson DJ, Schwager F, Pintard L, Gotta M, Goldstein B: A Single-Cell Biochemistry Approach Reveals PAR Complex Dynamics during Cell Polarization. Dev Cell 2017, 42:416-434.e11.

By analysing protein complexes in single embryos at different stages of the first cell cycle, Dickinson et al. show that PAR-3 forms large clusters during the polarity establishment phase to promote the localisation of the anterior PARs by advection. The cluster size decreases during the maintenance phase due to the phophorylation of the PAR-3 oligomerisation domain by Polo-like kinase.

25**. Rodriguez J, Peglion F, Martin J, Hubatsch L, Reich J, Hirani N, Gubieda AG, Roffey J, Fernandes AR, St Johnston D, et al.: aPKC Cycles between Functionally Distinct PAR Protein Assemblies to Drive Cell Polarity. Dev Cell 2017, 42:400-415.e9.

This work shows that PAR-6 and PKC-3 cycle between two distinct cortical complexes. During polarity establishment, they associate with PAR-3 clusters, which mediate their anterior localisation. During polarity maintenance, they associate with CDC42 to form diffusible complexes with active PKC-3

26*. Wang S-C, Low TYF, Nishimura Y, Gole L, Yu W, Motegi F: Cortical forces and CDC-42 control clustering of PAR proteins for Caenorhabditis elegans embryonic polarization. Nat Cell Biol 2017, 19:988-995.

This study presents evidence that cortical contractility promotes the clustering of PAR-3 to enhance the localisation of the anterior PARs by advection. 
27. Soriano EV, Ivanova ME, Fletcher G, Riou P, Knowles PP, Barnouin K, Purkiss A, Kostelecky B, Saiu P, Linch M, et al.: aPKC Inhibition by Par3 CR3 Flanking Regions Controls Substrate Access and Underpins ApicalJunctional Polarization. Dev Cell 2016, 38:384-398.

28. Nance J, Priess JR: Cell polarity and gastrulation in C. elegans. Development 2002, 129:387-397.

29. Nance J, Munro EM, Priess JR: C. elegans PAR-3 and PAR-6 are required for apicobasal asymmetries associated with cell adhesion and gastrulation. Development 2003, 130:5339-5350.

30. Anderson DC, Gill JS, Cinalli RM, Nance J: Polarization of the C. elegans embryo by RhoGAP-mediated exclusion of PAR-6 from cell contacts. Science 2008, 320:1771-1774.

31*. Klompstra D, Anderson DC, Yeh JY, Zilberman Y, Nance J: An instructive role for $\mathbf{C}$. elegans $\mathbf{E}$-cadherin in translating cell contact cues into cortical polarity. Nat Cell Biol 2015, doi:10.1038/ncb3168.

This study shows that C. elegans E-cadherin (HMR-1) plays an instructive role in blastomere polarity by recruiting the CDC42-GAP, PAC-1, to sites of cell contact, thereby restricting CDC42 activity to the free, apical surface.

32*. Koorman T, Klompstra D, van der Voet M, Lemmens I, Ramalho JJ, Nieuwenhuize S, van den Heuvel S, Tavernier J, Nance J, Boxem M: A combined binary interaction and phenotypic map of $\mathrm{C}$. elegans cell polarity proteins. Nat Cell Biol 2016, 18:337-346.

A large scale yeast two hybrid screen reveals that PAC-1 binds to PAR-6. This interaction is essential for blastomere polarisation.

33. Stephenson RO, Yamanaka Y, Rossant J: Disorganized epithelial polarity and excess trophectoderm cell fate in preimplantation embryos lacking $\mathrm{E}$ cadherin. Development 2010, 137:3383-3391.

34**. Korotkevich E, Niwayama R, Courtois A, Friese S, Berger N, Buchholz F, Hiiragi T: The Apical Domain Is Required and Sufficient for the First Lineage Segregation in the Mouse Embryo. Dev Cell 2017, 40:235-247.e7.

Single blastomeres isolated from the 8-cell mouse embryo form an apical domain without cell contact and divide asymmetrically to form one polarised trophectoderm cell and an apolar inner cell. Apolar cells can be induced to polarise by contact with an uncoated bead.

35. Bryant DM, Datta A, Rodriguez-Fraticelli AE, Peränen J, Martin-Belmonte F, Mostov KE: A molecular network for de novo generation of the apical surface and lumen. Nat Cell Biol 2010, 12:1035-1045.

36. Blasky AJ, Mangan A, Prekeris R: Polarized protein transport and lumen formation during epithelial tissue morphogenesis. Annu Rev Cell Dev Biol 2015, 31:575-591. 
37. Li D, Mangan A, Cicchini L, Margolis B, Prekeris R: FIP5 phosphorylation during mitosis regulates apical trafficking and lumenogenesis. $E M B O R e p$ 2014, 15:428-437.

38. Mrozowska PS, Fukuda M: Regulation of podocalyxin trafficking by Rab small GTPases in 2D and 3D epithelial cell cultures. J Cell Biol 2016, 213:355-369.

39**. Klinkert K, Rocancourt M, Houdusse A, Echard A: Rab35 GTPase couples cell division with initiation of epithelial apico-basal polarity and lumen opening. Nat Commun 2016, 7:11166.

Rab35 helps to specify the apical membrane initiation site (AMIS) in MDCK cell cysts by targeting apical vesicles to the site of cytokinesis through a direct interaction with the cytoplasmic tail of Podocalyxin.

40**. Mangan AJ, Sietsema DV, Li D, Moore JK, Citi S, Prekeris R: Cingulin and actin mediate midbody-dependent apical lumen formation during polarization of epithelial cells. Nat Commun 2016, 7:12426.

The tight junction protein, Cingulin, is recruited to the site of cytokinesis through an interaction with midbody microtubules and tethers Rab11 vesicles to promote AMIS formation.

41. Rodriguez-Boulan E, Macara IG: Organization and execution of the epithelial polarity programme. Nat Rev Mol Cell Biol 2014, 15:225-242.

42. St Johnston D, Ahringer J: Cell polarity in eggs and epithelia: parallels and diversity. Cell 2010, 141:757-774.

43**. Dong W, Zhang X, Liu W, Chen Y-J, Huang J, Austin E, Celotto AM, Jiang WZ, Palladino MJ, Jiang Y, et al.: A conserved polybasic domain mediates plasma membrane targeting of $\mathbf{L g l}$ and its regulation by hypoxia. $\mathrm{J}$ Cell Biol 2015, 211:273-286.

Lgl binds to plasma membrane lipids through a polybasic region that is inactivated by hypoxia. Phosphorylation of this region by aPKC neutralises its net positive charge and prevents lipid binding, providing a mechanism for Lgl cortical exclusion by aPKC.

44**. Bailey MJ, Prehoda KE: Establishment of Par-Polarized Cortical Domains via Phosphoregulated Membrane Motifs. Dev Cell 2015, 35:199-210.

This study shows that a number of proteins contain membrane-binding polybasic domains that are phosphorylated by aPKC to exclude them from the apical cortex, including Lgl, Miranda and Numb.

45. Martin-Belmonte F, Gassama A, Datta A, Yu W, Rescher U, Gerke V, Mostov $\mathrm{K}$ : PTEN-mediated apical segregation of phosphoinositides controls epithelial morphogenesis through Cdc42. Cell 2007, 128:383-397.

46**. Ruch TR, Bryant DM, Mostov KE, Engel JN: Par3 integrates Tiam1 and 
phosphatidylinositol 3-kinase signaling to change apical membrane identity. Mol Biol Cell 2017, 28:252-260.

Inducible mislocalisation of Par-3 to the apical cortex of MDCK cells leads to the recruitment of PI3-kinase and the Rac activator, TIAM1, triggering a change in domain identity.

47. Harris TJC, Peifer M: The positioning and segregation of apical cues during epithelial polarity establishment in Drosophila. J Cell Biol 2005, 170:813823.

48**. Ahmed SM, Macara IG: The Par3 polarity protein is an exocyst receptor essential for mammary cell survival. Nat Commun 2017, 8:14867.

A lysine-rich domain in Par-3 binds Exo7 to recruit the exocyst to tight junctions. This is important for the targeting of E-cadherin secretion.

49**. Jossin Y, Lee M, Klezovitch O, Kon E, Cossard A, Lien W-H, Fernandez TE, Cooper JA, Vasioukhin V: Llgl1 Connects Cell Polarity with Cell-Cell Adhesion in Embryonic Neural Stem Cells. Dev Cell 2017, 41:481-495.e5.

LLGL1 binds to the C-terminal tail of $\mathrm{N}$-cadherin to induce its internalisation from the lateral membrane. This is essential for $\mathrm{N}$-cadherin enrichment at adherens junctions.

50*. Zihni C, Vlassaks E, Terry S, Carlton J, Leung TKC, Olson M, Pichaud F, Balda MS, Matter K: An apical MRCK-driven morphogenetic pathway controls epithelial polarity. Nat Cell Biol 2017, 9:833.

Apical CDC42-GTP activates MRCK to promote Myosin contractility. This drives the separation of PAR- 6 and aPKC from Par-3 and promotes the morphogenesis of the apical domain.

51. Siller KH, Doe CQ: Spindle orientation during asymmetric cell division. Nat Cell Biol 2009, 11:365-374.

52**. Inaba M, Venkei ZG, Yamashita YM, Banerjee U: The polarity protein Baz forms a platform for the centrosome orientation during asymmetric stem cell division in the Drosophila male germline. Elife 2015, 4:e04960.

Bazooka (Par-3) orients the mitotic spindle in Drosophila male germline stem cells by recruiting one centrosome to sites of adhesion between the stem cell and the Hub. Mitosis is delayed in cells with misoriented centrosomes by a centrosome docking checkpoint that depends on the phosphorylation of Bazooka by Par-1.

53. Jiang T, McKinley RFA, McGill MA, Angers S, Harris TJC: A Par-1-Par-3Centrosome Cell Polarity Pathway and Its Tuning for Isotropic Cell Adhesion. Curr Biol 2015, 25:2701-2708.

54. Besson C, Bernard F, Corson F, Rouault H, Reynaud E, Keder A, Mazouni K, Schweisguth F: Planar Cell Polarity Breaks the Symmetry of PAR Protein Distribution prior to Mitosis in Drosophila Sensory Organ Precursor Cells. 
Curr Biol 2015, 25:1104-1110.

$55^{* *}$. Banerjee JJ, Aerne BL, Holder MV, Hauri S, Gstaiger M, Tapon N: Meru couples planar cell polarity with apical-basal polarity during asymmetric cell division. Elife 2017, 6:e25014.

This work identifies the RASSF protein Meru as a cell-type specific adaptor that couples Bazooka localisation to the planar cell polarity system in Drosophila sensory organ precursors.

56. Bergstralh DT, Lovegrove HE, St Johnston D: Discs large links spindle orientation to apical-basal polarity in Drosophila epithelia. Curr Biol 2013, 23:1707-1712.

57. Saadaoui M, Machicoane M, di Pietro F, Etoc F, Echard A, Morin X: Dlg1 controls planar spindle orientation in the neuroepithelium through direct interaction with LGN. $J$ Cell Biol 2014, 206:707-717.

58. Bergstralh DT, Lovegrove HE, Kujawiak I, Dawney NS, Zhu J, Cooper S, Zhang R, St Johnston D: Pins is not required for spindle orientation in the Drosophila wing disc. Development 2016, 143:2573-2581.

59. Bosveld F, Markova O, Guirao B, Martin C, Wang Z, Pierre A, Balakireva M, Gaugue I, Ainslie A, Christophorou N, et al.: Epithelial tricellular junctions act as interphase cell shape sensors to orient mitosis. Nature 2016, 530:495498.

60. Bornens M: The Centrosome in Cells and Organisms. Science 2012, 335:422-426.

61. Hong E, Jayachandran P, Brewster R: The polarity protein Pard3 is required for centrosome positioning during neurulation. Dev Biol 2010, 341:335-345.

62. Feldman JL, Priess JR: A role for the centrosome and PAR-3 in the handoff of MTOC function during epithelial polarization. Curr Biol 2012, 22:575-582.

63**. Burute M, Prioux M, Blin G, Truchet S, Letort G, Tseng Q, Bessy T, Lowell S, Young J, Filhol O, et al.: Polarity Reversal by Centrosome Repositioning Primes Cell Scattering during Epithelial-to-Mesenchymal Transition. Dev Cell 2017, 40:168-184.

At an early stage of EMT, centrosomes move from the apical surface of epithelial cells towards the cell centre. This correlates with their acquisition of mesenchymal properties and cell migration.

64. Wu J, Akhmanova A: Microtubule-Organizing Centers. Annu Rev Cell Dev Biol 2017, doi:10.1146/annurev-cellbio-100616-060615.

65. Wang S, Wu D, Quintin S, Green RA, Cheerambathur DK, Ochoa SD, Desai A, Oegema K: NOCA-1 functions with $\gamma$-tubulin and in parallel to Patronin to assemble non-centrosomal microtubule arrays in C. elegans. Elife 2015, 
4:e08649.

66. Goldspink DA, Rookyard C, Tyrrell BJ, Gadsby J, Perkins J, Lund EK, Galjart $\mathrm{N}$, Thomas $\mathrm{P}$, Wileman T, Mogensen MM: Ninein is essential for apico-basal microtubule formation and CLIP-170 facilitates its redeployment to noncentrosomal microtubule organizing centres. Open Biology 2017, 7:160274.

67. Toya M, Kobayashi S, Kawasaki M, Shioi G, Kaneko M, Ishiuchi T, Misaki K, Meng W, Takeichi M: CAMSAP3 orients the apical-to-basal polarity of microtubule arrays in epithelial cells. Proc Natl Acad Sci USA 2016, 113:332-337.

68*. Khanal I, Elbediwy A, Diaz de la Loza MDC, Fletcher GC, Thompson BJ: Shot and Patronin polarise microtubules to direct membrane traffic and biogenesis of microvilli in epithelia. J Cell Sci 2016, 129:2651-2659.

The apical spectrin cytoskeleton plays a role in recruiting Shot and Patronin to the apical cortex of the epithelial follicle cells, where they organise the apical-basal array of microtubules.

69**. Nashchekin D, Fernandes AR, St Johnston D: Patronin/Shot cortical foci assemble the noncentrosomal microtubule array that specifies the Drosophila anterior-posterior axis. Dev Cell 2016, 38:61-72.

Par-1 defines the Drosophila anterior-posterior axis by excluding Shot and Patronin from the posterior cortex of the oocyte. Shot/Patronin complexes along the anterior/lateral cortex acts as noncentrosomal microtubule organising centres, resulting in the formation of the polarised microtubule network that directs the localisation of the axis determinants, bicoid and oskar mRNAs.

70. Noordstra I, Liu Q, Nijenhuis W, Hua S, Jiang K, Baars M, Remmelzwaal S, Martin M, Kapitein LC, Akhmanova A: Control of apico-basal epithelial polarity by the microtubule minus-end-binding protein CAMSAP3 and spectraplakin ACF7. J Cell Sci 2016, 129:4278-4288.

71. Nakanishi Y, Reina-Campos M, Nakanishi N, Llado V, Elmén L, Peterson S, Campos A, De SK, Leitges M, Ikeuchi H, et al.: Control of Paneth Cell Fate, Intestinal Inflammation, and Tumorigenesis by PKC $\lambda /$ ı. Cell Rep 2016, 16:3297-3310.

72. Zheng W, Umitsu M, Jagan I, Tran CW, Ishiyama N, BeGora M, Araki K, Ohashi PS, Ikura M, Muthuswamy SK: An interaction between Scribble and the NADPH oxidase complex controls M1 macrophage polarization and function. Nat Cell Biol 2016, 18:1244-1252. 
Figures:
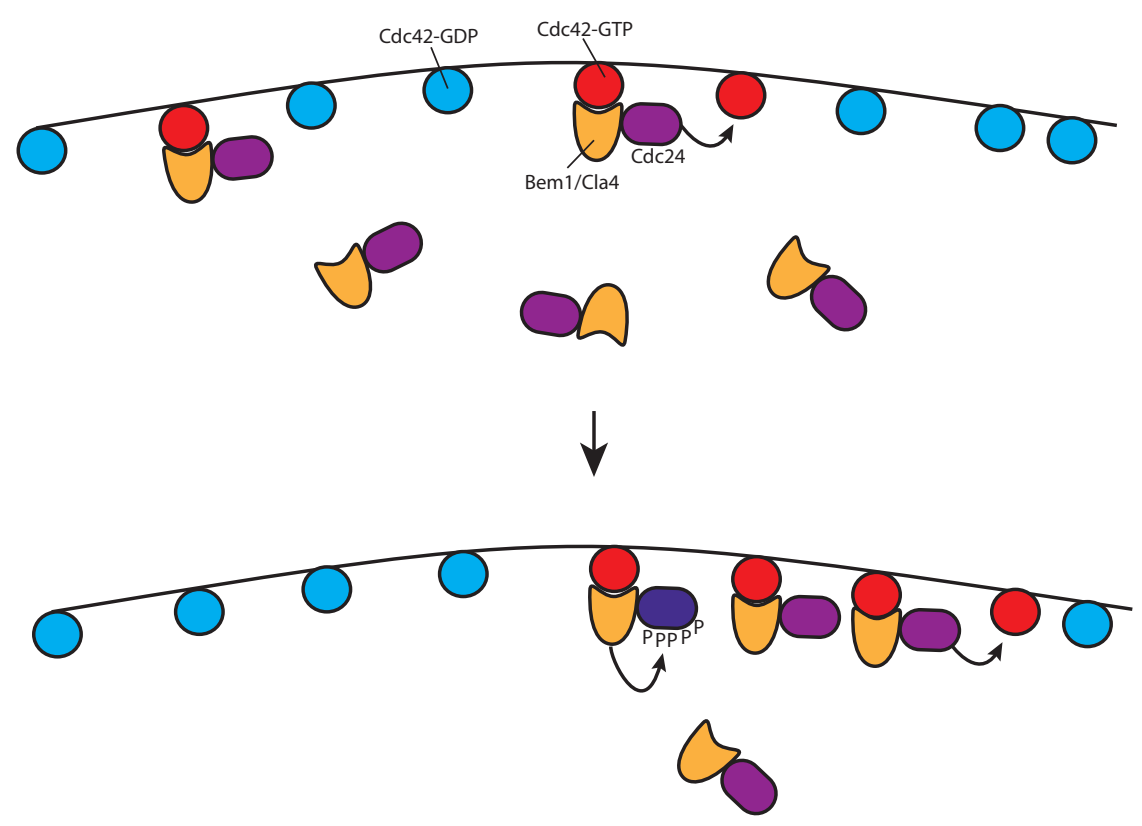

Figure 1. Establishment of a single stable polarity site in Saccharomyces cerevisiae depends on positive and negative feedback and competition between sites for limited cytosolic factors. Bem1/Cla4 binding to active CDC42-GTP recruits the CDC42 Guanine nucleotide exchange factor (GEF), CDC24, to activate more CDC42. The $\mathrm{p} 21$-activated kinase Cla4 limits this process by phosphorylating and inactivating CDC24. As the focus of active CDC42 expands, it competes with other foci for limited amounts of these activators. 


\section{Polarity Establishment}
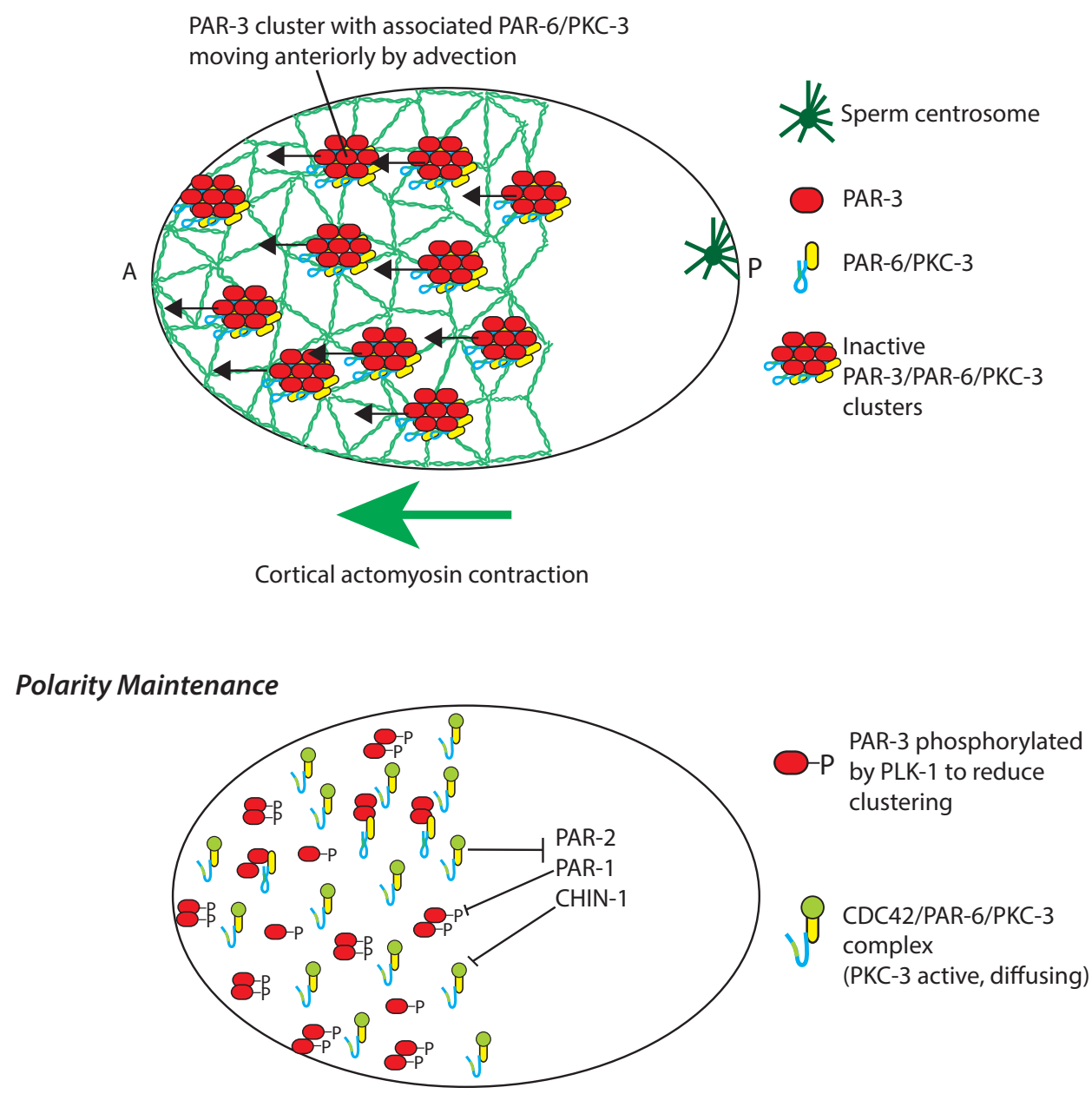

Figure 2. Polarity establishment and maintenance in C. elegans. Top panel:

Symmetry is broken at fertilisation when microtubules nucleated from the sperm centrosome contact the posterior cortex to trigger a contraction of the actomyosin cortex towards the anterior. At this stage, PAR-3 oligomerises to form clusters that efficiently recruit PAR-6 and PKC-3. The clusters are carried by advection to the anterior by the cortical flows to establish the anterior PAR domain. Lower panel: Polo-like kinase phosphorylates PAR-3 to reduce its 
oligomerisation during the maintenance phase. This allows PAR-6 and PKC-3 to associate with CDC42-GTP to form a diffusible complex in which PKC-3 is active. Active PKC-3 maintains polarity by inhibiting PAR-2, PAR-1 and CHIN-1 (possibly indirectly) at the boundary between the anterior and posterior domains. This is counteracted on the posterior side by phosphorylation of PAR-3 by PAR-1 and the inactivation of CDC42 by CHIN1.

A

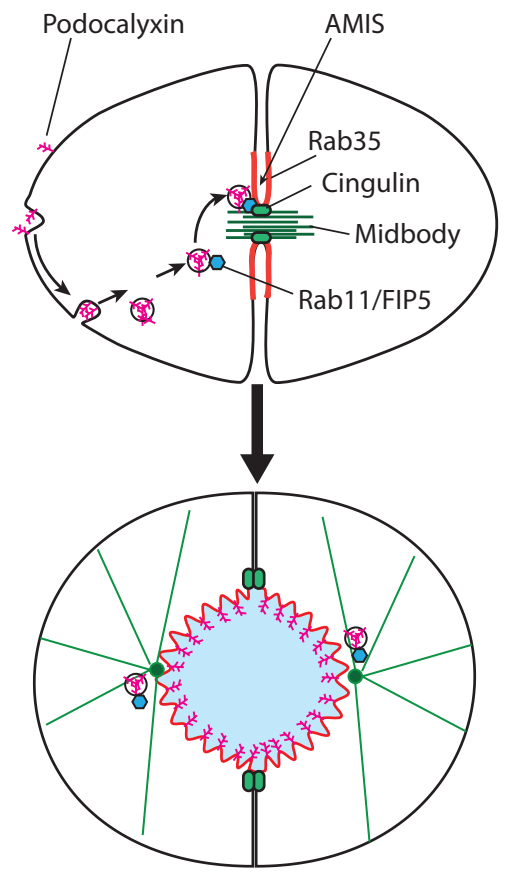

B

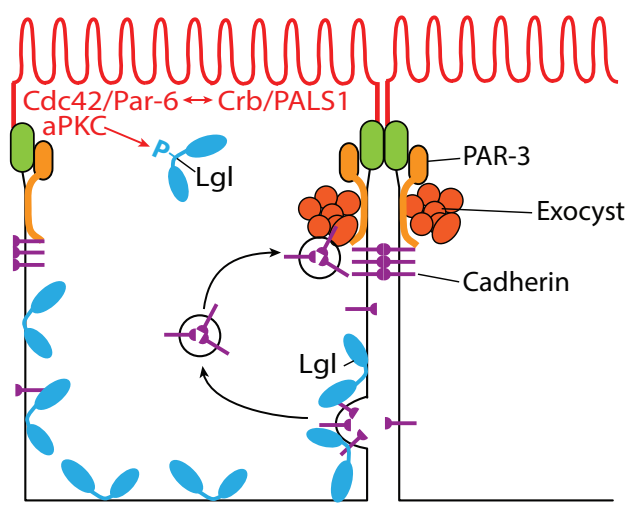

Figure 3.

A) Polarity establishment in MDCK-cell cysts. Apical proteins, such as Podocalyxin, localise around the entire surface of single MDCK cells in 3D culture. When the cells divide, apical factors are endocytosed from the plasma membrane and are recycled to the apical membrane initiation site (AMIS), which forms at the site of cytokinesis. The polarised secretion of these apical vesicles at the AMIS depends on an interaction between Podocalyxin and Rab35 and the recruitment of Rab11/FIP5 positive vesicles by Cingulin, which associates with midbody microtubules. Secretion of Podocalyxin at the AMIS promotes the formation of an apical lumen between the two daughter cells.

B) A model for Cadherin targeting to Adherens junctions. Lgl (LLGL1) is restricted to the basolateral membrane domain by apical aPKC, which phosphorylates the hinge region of Lgl to prevent its binding to membrane phospholipids. In the neural stem cells of the developing mouse brain, binding of LLGL1 to the cytoplasmic tail of N-cadherin triggers its endocytosis from the lateral membrane and recycling to sub-apical Adherens junctions. In MDCK cells, E-cadherin exocytosis is targeted to the junctional domain by the Exocyst, which is recruited to junctions by the binding of Exo70 to the Lysine rich C-terminal domain of Par-3. This model assumes that N-cadherin in neural stem cells and E-cadherin in MDCK cells are trafficked by the same mechanisms, which has not yet been tested. 

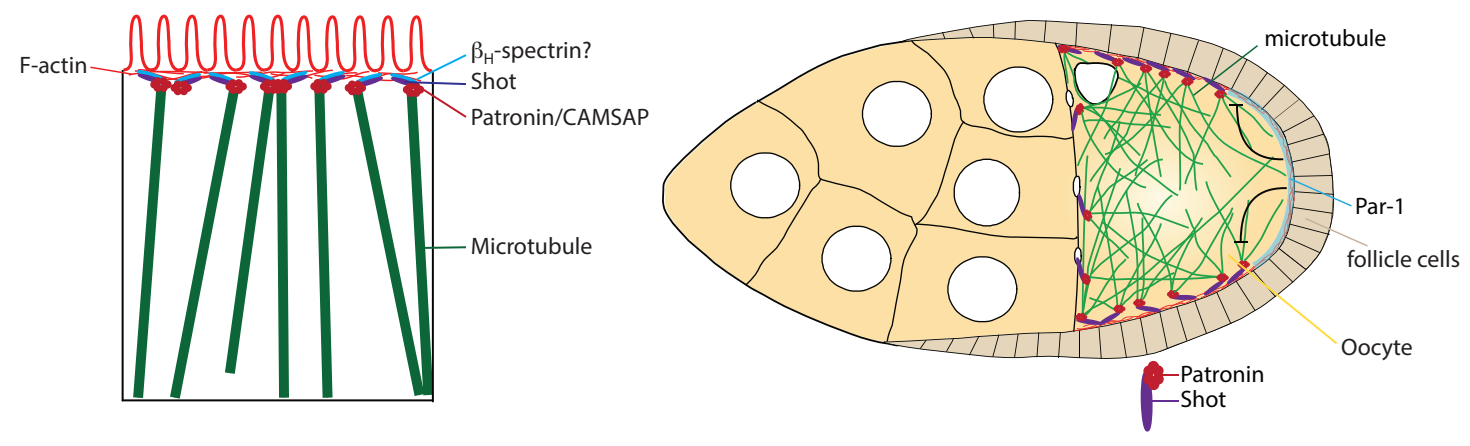

Figure 4. Polarisation of the microtubule cytoskeleton

A) In many postmitotic epithelial cells, microtubules grow from noncentrosomal microtubule organising centres (ncMTOCs) along the apical cortex that contain the spectraplakin, Shortstop (Shot; ACF7 in mammals) and the microtubule minus-end binding protein, Patronin (CAMSAP2 and 3 in mammals), but not $\gamma$-tubulin. These ncMTOCs are thought to function by capturing and stabilising microtubule minus ends that can then act as templates for microtubule growth to set up an apical-basal array of microtubules. It has been proposed that the ncMTOCs are recruited to the apical cortex of Drosophila follicle cells by the apical spectrin cytoskeleton, which is composed of $\beta_{\mathrm{H}}$-spectrin $/ \alpha$-spectrin tetramers.

B) The Drosophila anterior-posterior axis becomes polarised in response to a signal from the posterior follicle cells that induces the localisation of Par-1 to the posterior cortex of the oocyte. Par-1 excludes Shot/Patronin ncMTOCs from the posterior cortex, resulting in the formation of a polarised microtubule network that grows from ncMTOCs along the anterior/lateral cortex. This polarised microtubule cytoskeleton then directs the transport of bicoid and oskar mRNAs to opposite poles of the oocyte to define the anterior- posterior axis of the future embryo. 